\title{
Hierarchical solutions of the Sherrington-Kirkpatrick model: Exact asymptotic behavior near the critical temperature
}

\author{
V. Janiš and A. Klíc \\ Institute of Physics, Academy of Sciences of the Czech Republic, \\ $\mathrm{Na}$ Slovance 2, CZ-18221 Praha, Czech Republic
}

(Dated: November 13, 2018)

\begin{abstract}
We analyze the replica-symmetry-breaking construction in the Sherrington-Kirkpatrick model of a spin glass. We present a general scheme for deriving an exact asymptotic behavior near the critical temperature of the solution with an arbitrary number of discrete hierarchies of the broken replica symmetry. We show that all solutions with finite-many hierarchies are unstable and only the scheme with infinite-many hierarchies becomes marginally stable. We show how the solutions from the discrete replica-symmetry-breaking scheme go over to the continuous one with increasing the number of hierarchies.

PACS numbers: 64.60.Cn,75.50.Lk
\end{abstract}

\section{INTRODUCTION}

Spin glasses are specific unusual statistical systems, since except for a few limiting cases no exact analytic solutions are available, even for mean-field models. A formal exact solution of the mean-field Ising spin glass, Sherrington-Kirkpatrick (SK) model, is known in form of the replica-symmetry-breaking (RSB) scheme of Parisi. 1] The solution of the RSB construction is, however, explicitly known only approximately. One can iterate the RSB scheme with finite many hierarchies. In this way $1 \mathrm{RSB}$ and $2 \mathrm{RSB}$ solutions were explicitly calculated. 2] Or one can go over to the limit of infinite number of hierarchies and try to find a solution to the continuous limit of the RSB scheme controlled by a nonlinear partial differential equation. A solution to the continuous RSB scheme is known near the critical temperature. 1, 3, 4, 5, 6] Recently, a numerical solution of the differential equation from the continuous RSB scheme was obtained also beyond the critical region at zero magnetic field. [7]

The RSB construction has hence presently two analytically accessible limits: the discrete scheme with a few hierarchies and the continuous limit. The former, within 1RSB or 2RSB schemes, can be applied anywhere in the low-temperature phase. The latter, on the other hand, is viable practically only near the critical temperature at zero magnetic field. Originally the discrete scheme was considered as an approximation to the ultimate solution, the continuous limit. Recently, however, variants of mean-field spin glass models such as random-energy [8], $p$-spin [9] or Potts [10], display regions where already 1RSB scheme becomes stable. The discrete RSB scheme has thus won its own substantiation beyond a mere approximate scheme. Moreover, using the concept of thermodynamic homogeneity one can derive the discrete RSB scheme with finite-many hierarchies without any specific assumptions on the behavior of the order parameters. 11] Even in the limiting case of the discrete RSB scheme with infinite-many hierarchies one need not end up with the continuous limit. To derive the continuous limit one has to assume that both differences $\Delta q_{i}=q_{i+1}-q_{i}$ and $\Delta m_{i}=m_{i}-m_{i+1}$ from the Parisi construction are infinitesimal (of order $1 / K$ ) if the number of hierarchies $K$ approaches infinity. There is no á priori reason for such a uniform behavior and unless proved by explicit calculations, it must be assumed as an ansatz, as actually done by Parisi in his derivation of the full RSB solution.

The number of hierarchies used in the RSB solution is generally a free parameter that cannot be derived from free energy. This number is determined from stability of the thermodynamic equilibrium state. To decide how many hierarchies in the RSB scheme are needed to reach a thermodynamically stable solution and whether the infinite number of hierarchies leads to the continuous distribution of the order parameters one has to analyze the RSB scheme with a variable number of hierarchies. This is possible only in specific asymptotic limits such as the asymptotic region near the critical temperature. The existing analyses [1, 3, 4, 5, 6] of the RSB scheme near the critical temperature use an incomplete expansion of free energy where only a single, simplest term from the highest-order contribution is taken into consideration. Such an expansion does not reproduce correctly the asymptotic behavior of the RSB solutions with a few hierarchies (1RSB, 2RSB). It hence cannot serve as a proof of validity of the continuous limit for the RSB solution with infinite-many hierarchies.

The aim of this paper is to present a general scheme for analyzing the full discrete hierarchy of RSB solutions for mean-field models of spin glasses. We in particular concentrate on the SK model at zero magnetic field and use the asymptotic solution to determine the way in which the limit of infinite many hierarchical levels is approached. With the exact asymptotic solution for arbitrary numbers of RSB hierarchies we prove that the stable solution of the SK model is the continuous limit of the RSB scheme. We explicitly evaluate the equations for the order parameters in the first nontrivial order of the expansion parameter $\theta=1-T / T_{c}$ around the critical temperature $T_{c}$. We perform the expansion generally for the RSB solution with $K$ hierarchies. To achieve 
this goal, we use an explicit representation for the hierarchical free energy (partition function) with $K$ levels from which we derive equations for the order parameters. These equations are then asymptotically expanded near the critical temperature to obtain an explicit leadingorder asymptotic behavior of all the order parameters at any hierarchical level of the RSB construction. From it we reconstruct the continuous version of the RSB scheme when the number of hierarchies is limited to infinity. We explicitly show that any solution with a finite number of hierarchies is unstable even arbitrarily close to the critical temperature and only the continuous limit, as expected, becomes marginally stable in the spin-glass phase. [4]

\section{RSB SOLUTION WITH $K$ HIERARCHIES}

We avoid the replica trick and use the explicit representation of the RSB solution with generally $K$ hierarchies from Ref. 11] derived from the thermodynamic approach of Thouless, Anderson, and Palmer. In this representation the solution has $2 K+1$ order parameters. There are $K+1$ physical ones, $q, \Delta \chi_{1}, \ldots, \Delta \chi_{K}$ related to various ways squares of local magnetizations can be calculated. The other $K$ parameters, $m_{1}, \ldots, m_{K}$, have a geometric origin and are connected with the way we break the replica symmetry.

To derive an analytic representation for the $K$-level hierarchical free energy we start with an averaged freeenergy density of the Sherrington-Kirkpatrick model with $\nu$ real replicas

$$
\begin{gathered}
f_{\nu}=\frac{\beta J^{2}}{4}\left[\frac{1}{\nu} \sum_{a \neq b}^{\nu}\left\{\left(\chi^{a b}\right)^{2}+2 q \chi^{a b}\right\}-(1-q)^{2}\right] \\
-\frac{1}{\beta \nu} \int_{-\infty}^{\infty} \frac{d \eta}{\sqrt{2 \pi}} e^{-\eta^{2} / 2} \ln \operatorname{Tr}_{S} \exp \left\{\beta^{2} J^{2} \sum_{a<b}^{\nu} \chi^{a b} S^{a} S^{b}\right. \\
\left.+\beta \bar{h} \sum_{a=1}^{\nu} S^{a}\right\}
\end{gathered}
$$

where the trace $\operatorname{Tr}_{S}$ runs over the replicated Ising spins $S^{a}= \pm 1$. We denoted the internal magnetic field $\bar{h}=h+\eta \sqrt{q}$. Averaging over the fluctuating internal field $\eta$ replaces averaging over the spin exchange $J_{i j}$ in the mean-field solution. The averaged order parameter at the saddle point $q=\left\langle\left\langle S^{a}\right\rangle_{T}^{2}\right\rangle_{a v}$ does not depend on the replica index due to equivalence of replicas. Real replicas allow us to introduce local inter-replica susceptibilities as order parameters being at the saddle point $\chi^{a b}=\left\langle\left\langle S^{a} S^{b}\right\rangle_{T}\right\rangle_{a v}-q$. Angular brackets \langle\rangle$_{T}$ denote averaging over the configurations of replicated spins.

The hierarchical solution is constructed by successive applications of the replica symmetry ansatz on the matrix of the overlap susceptibilities We remind that $\chi^{a a}=0$. For $K=1$ we choose $\chi^{a \neq b}=\chi_{1}$ and decouple the spin variables via a Hubbard-Stratonovich transformation and a new fluctuating replica-diagonal field $\lambda_{1}$. This transformation enables us to evaluate the trace over the replicated spins explicitly. We recover 1RSB solution of Parisi. The next hierarchy is obtained if we replace each matrix element $\chi^{a b}$ by a matrix $\chi_{\alpha \beta}^{a b}$. We again apply the replica symmetry ansatz on non-diagonal elements. We have two parameters $\chi_{\alpha \neq \beta}^{a a}=\chi_{1}$ and $\chi_{\alpha \beta}^{a \neq b}=\chi_{2}$. The replicated spins will be decoupled with two HubbardStratonovich fluctuating field $\lambda_{1}, \lambda_{2}$. We proceed in this hierarchical construction up to the desired $K$-level solution.

We can conveniently represent the $K$-level free energy recursively. We define a sequence of partition functions

$$
Z_{l}=\left[\int_{-\infty}^{\infty} \mathcal{D} \lambda_{l} Z_{l-1}^{m_{l}}\right]^{1 / m_{l}}
$$

where we used an abbreviation for the Gaussian differential $\mathcal{D} \lambda_{l} \equiv \mathrm{d} \lambda_{l} e^{-\lambda_{l}^{2} / 2} / \sqrt{2 \pi}$. The initial condition reads $Z_{0}=\cosh \left[\beta\left(h+\eta \sqrt{q}+\sum_{l=1}^{K} \lambda_{l} \sqrt{\Delta \chi_{l}}\right)\right]$. The averaged free energy density with $K$ hierarchies can then be represented as [1]

$$
\begin{aligned}
f^{K}\left(q, \Delta \chi_{1}, \ldots, \Delta \chi_{K} ; m_{1}, \ldots, m_{K}\right)=-\frac{1}{\beta} \ln 2 \\
+\frac{\beta}{4} \sum_{l=1}^{K} m_{l} \Delta \chi_{l}\left[2\left(q+\sum_{i=l+1}^{K} \Delta \chi_{i}\right)+\Delta \chi_{l}\right] \\
-\frac{\beta}{4}\left(1-q-\sum_{l=1}^{K} \Delta \chi_{l}\right)^{2}-\frac{1}{\beta} \int_{-\infty}^{\infty} \mathcal{D} \eta \ln Z_{K}
\end{aligned}
$$

with order parameters $q, \Delta \chi_{l}$ and $m_{l}, l=1, \ldots, K$ to be determined from stationarity equations.

To represent the mean-field equations we introduce a set of hierarchical density matrices in the space of fluctuating random fields $\lambda_{l}$. We define $\rho_{l}\left(\eta, \lambda_{K}, \ldots, \lambda_{l}\right)=$ $Z_{l-1}^{m_{l}} /\left\langle Z_{l-1}^{m_{l}}\right\rangle_{\lambda_{l}}$. We further introduce short-hand notations $t \equiv \tanh \left[\beta\left(h+\eta \sqrt{q}+\sum_{l=1}^{K} \lambda_{l} \sqrt{\Delta \chi_{l}}\right)\right]$ and $\langle t\rangle_{l}\left(\eta, \lambda_{K}, \ldots, \lambda_{l+1}\right) \equiv\left\langle\rho_{l} \ldots\left\langle\rho_{1} t\right\rangle_{\lambda_{1}} \ldots\right\rangle_{\lambda_{l}}$ with $\left\langle X\left(\lambda_{l}\right)\right\rangle_{\lambda_{l}} \equiv \int_{-\infty}^{\infty} \mathcal{D} \lambda_{l} X\left(\lambda_{l}\right)$.

With the above definitions we can write down the stationarity equations for the physical order parameters

$$
\begin{aligned}
q^{K} & =\left\langle\langle t\rangle_{K}^{2}\right\rangle_{\eta} \\
\Delta \chi_{l}^{K} & =\left\langle\left\langle\langle t\rangle_{l-1}^{2}\right\rangle_{K}\right\rangle_{\eta}-\left\langle\left\langle\langle t\rangle_{l}^{2}\right\rangle_{K}\right\rangle_{\eta}
\end{aligned}
$$

and for the geometric ones

$$
m_{l}^{K}=\frac{4}{\beta^{2}} \frac{\left\langle\left\langle\ln Z_{l-1}\right\rangle_{K}\right\rangle_{\eta}-\left\langle\left\langle\ln Z_{l}\right\rangle_{K}\right\rangle_{\eta}}{\left\langle\left\langle\langle t\rangle_{l-1}^{2}\right\rangle_{K}\right\rangle_{\eta}^{2}-\left\langle\left\langle\langle t\rangle_{l}^{2}\right\rangle_{K}\right\rangle_{\eta}^{2}}
$$

where index $l=1, \ldots, K$. Note that there is a direct connection to the order parameters of the Parisi $K$-level RSB solution. The Parisi parameters are identified as follows $q=q^{K}$ and $q_{l}=q^{K}+\sum_{i=l}^{K} \Delta \chi_{i}^{K}$. 
It is important to stress that the hierarchical freeenergy density from Eq. (3) is not identical with discrete $K$-level RSB solutions used in the literature to analyze the critical region of the spin-glass transition in the SK model [3, 4, 5, 6]. The latter functionals can be derived from the asymptotic limit $T \nearrow T_{c}$ of Eq. (3) by using $K^{-1}$ expansion to order $O\left(K^{-1}\right)$ with an ansatz $\Delta \chi_{l}=\Delta_{l} / K$ and $m_{l} / m_{l+1}=1+\delta_{l} / K$. This step is in fact a discrete version of the continuous limit studied in Sec. D] The full discrete RSB free energy equivalent to Eq. (3) was derived in Ref. 12] but not used in explicit calculations. A necessity of modifications to the $K$-level hierarchical free energies from Refs. 3, 4, 5, 6]. when $K$ remains finite was discussed in Ref. 13].

The hierarchical free energy, Eq. (3), and the respective stationarity equations, Eq. (44) and Eq. (5), were derived within the real-replica approach. The real replicas simulate dependence of the mean-field solutions on initial conditions. The RSB scheme says that the initial configurations of the order parameters are ordered hierarchically according to the strength with which they influence the resulting equilibrium state. The instantaneous value of the local magnetization is $\tanh \left[\beta\left(h+\eta \sqrt{q}+\sum_{l=1}^{K} \lambda_{l} \sqrt{\Delta \chi_{l}}\right)\right]$, where the quenched random field $\eta$ stands for an actual configuration of spin couplings and the annealed random fields $\lambda_{l}$ for initial configurations of the spin variables from the $l$ th level with $l=1,2, \ldots, K$. The strength with which the $l$ th level influences the final equilibrated state is $\Delta \chi_{l}$. The density matrices $\rho_{l}$ express probability (weight) of the $l$ th level initial configurations in the final state. Hence, $\langle t\rangle_{l}$ denotes a local magnetization obtained after averaging over the first $l$ levels of initial configurations. The physical order parameters are then constructed from various possibilities to interchange making square and averaging over the hierarchies of the initial configurations of local magnetizations.

The number of hierarchical levels $K$ is a free parameter in the above construction. Its physical value leading to a unique physical solution is determined as the smallest number needed to reach a thermodynamically stable equilibrium state independent of initial conditions. Thermodynamic stability of a construction with $K$ hierarchies is determined from a set of $K+1$ stability conditions. 11] If

$$
\begin{aligned}
\Lambda_{l}=\beta^{2}\langle & \left\langle\left\langle 1-t^{2}\right.\right. \\
& \left.\left.\left.+\sum_{i=1}^{l} m_{i}\left(\langle t\rangle_{i-1}^{2}-\langle t\rangle_{i}^{2}\right)\right\rangle_{l}^{2}\right\rangle_{K}\right\rangle_{\eta} \geq 0 .
\end{aligned}
$$

are obeyed for $l=0,1, \ldots, K$, then the solution with $K$ hierarchies describes a locally stable equilibrium state. If one or more stability conditions are broken, we have to increase the number of hierarchies in our construction. With increasing the number of hierarchies used one has to observe a successive suppression of instabilities (negativeness of parameters $\Lambda_{l}$ ) when the construction con- verges. Only a convergent scheme can lead to a stable solution for some finite or, in the extreme case, infinite number of hierarchies.

\section{ASYMPTOTIC EXPANSION NEAR THE CRITICAL TEMPERATURE}

The order parameters in the spin-glass phase of the general $K$-level free energy from Eq. (3) cannot be solved explicitly unless we resort to solutions with only a few hierarchical levels $(K=1,2)$. The only chance to analyze the behavior of the entire hierarchical construction, that is with arbitrary numbers of hierarchies, is to expand the solution near the critical point where the order parameters are small. The hierarchical solution behaves in an external magnetic field differently from the rotationally invariant case. The physical order parameter $q$ and the geometric order parameters $m_{l}$ remain finite at the transition line and only the differences of the overlap susceptibilities $\Delta \chi_{l}$ are the genuine small parameters controlling the expansion near the critical point. While in the case of zero magnetic field all order parameters determining the stationarity points of free energy asymptotically vanish at the critical temperature. The latter case is on one hand more degenerate than the former, but on the other hand it is more symmetric with less expansion terms (only terms with even parity contribute in the rotationally invariant case). In this paper we will analyze only the rotationally invariant case, $h=0$.

The strategy to solve the stationarity equations for the hierarchical free energy asymptotically near the critical temperature is to expand the partition function into powers of the small order parameters and restrict the solution only to a functional subspace generated by a fixed polynomial expansion. We first use sch an expansion to derive the leading asymptotic limit of equations for the physical parameters $q, \Delta \chi_{l}$. At this stage we do not need to assume smallness of the geometric parameters. Smallness of $m_{l}, l=1, \ldots, K$ at zero magnetic field will be utilized later on when deriving the asymptotic form of mean-field equations for them.

It appears that it is sufficient to expand the stationarity equations for $q$ and $\Delta \chi_{l}$ only to the third order in these parameters. To control the expansion we introduce an auxiliary parameter $x$ that will be set to unity at the end. We hence write a canonical representation for the $l$ th partition sum

$$
\begin{aligned}
Z_{l-1}= & a_{00}^{(l)}+a_{02}^{(l)}\left(Y_{l+1}+c_{l} \lambda_{l}\right)^{2} x^{2}+a_{04}^{(l)}\left(Y_{l+1}\right. \\
& \left.+c_{l} \lambda_{l}\right)^{4} x^{4}+a_{06}^{(l)}\left(Y_{l+1}+c_{l} \lambda_{l}\right)^{6} x^{6}+x^{2}\left[a_{20}^{(l)}\right. \\
+ & \left.a_{22}^{(l)}\left(Y_{l+1}+c_{l} \lambda_{l}\right)^{2} x^{2}+a_{24}^{(l)}\left(Y_{l+1}+c_{l} \lambda_{l}\right)^{4} x^{4}\right] \\
& +x^{4}\left[a_{40}^{(l)}+a_{42}^{(l)}\left(Y_{l+1}+c_{l} \lambda_{l}\right)^{2} x^{2}\right]+x^{6} a_{60}^{(l)}
\end{aligned}
$$

where we denoted $Y_{l}=c \eta+\sum_{i=l}^{K} c_{i} \lambda_{i}, c_{l}=\beta \sqrt{\Delta \chi_{l}}, c=$ $\beta \sqrt{q}$. 
We now insert Eq. (7) into Eq. (2) and perform the integral over the fluctuating field $\lambda_{l}$. The result will again be reduced to a sixth order polynomial in $x$. Thereby we win recursive relations for the expansion parameters. We used the program MATHEMATICA to evaluate the coefficients. The coefficients needed in expansion (77) are listed in Appendix A.

Using the asymptotic expansion for the partition functions $Z_{l}$ we can explicitly evaluate products of the density matrices $\rho_{i}$. We will need to know the products within our precision only as quadratic polynomials in $x$. We explicitly obtain

$$
\prod_{i=k}^{l} \rho_{i}=1+\frac{x^{2}}{2} \sum_{i=k}^{l} m_{i} c_{i}\left[2 \lambda_{i} Y_{i+1}+c_{i}\left(\lambda_{i}^{2}-1\right)\right] .
$$

We use Eq. (8) in the defining equations for the physical order parameters. We denote $X_{l}=\sum_{i=1}^{l} \Delta \chi_{i}$, $Q=q+X_{K}$, and $M_{l}=\sum_{i=1}^{l} m_{i} \Delta \chi_{i}$ and obtain $(x=1)$

$$
q=\beta^{2} q\left\{1-2 \beta^{2} Q+\beta^{4}\left[\frac{2}{3} q^{2}+5 Q^{2}+2 M_{K}\right]\right\} .
$$

We skip the upper index $K$ at the order parameters in order not to make the notation cumbersome The above equation can further be rewritten to a more suitable form

$$
\begin{aligned}
& q=\beta^{2} q\left\{1-2 \beta^{2} Q+\frac{\beta^{4}}{3}\left[2 X_{K}^{2}-4 Q X_{K}\right.\right. \\
&+\left.\left.17 Q^{2}+6 M_{K}\right]\right\} .
\end{aligned}
$$

We note that $Q$ has the meaning of the EdwardsAnderson parameter. It is, however, not an independent order parameter.

Analogously we derive the asymptotic form of the defining equations for the differences of the overlap susceptibilities. We derive the following asymptotic equations

$$
\begin{gathered}
\Delta \chi_{l}=\beta^{2} \Delta \chi_{l}\left\{1-2 \beta^{2} Q+\beta^{4}\left[\frac{2}{3} \Delta \chi_{l}^{2}+2\left(Q-X_{l-1}\right)^{2}\right.\right. \\
\left.\left.+5 Q^{2}-\left(2 Q-2 X_{l-1}-m_{l}\right) \Delta \chi_{l}+2 M_{l-1}\right]\right\} .
\end{gathered}
$$

Notice that to determine the leading asymptotic behavior of $q$ and $\Delta \chi_{l}$ we had to keep first three orders in the expansion of the right-hand sides of the stationarity equations (4). Second-order term is degenerate in these equations and we obtain from it only the leading asymptotic behavior of the Edwards-Anderson parameter $Q$. Going to the third order then means that we have to calculate simultaneously to the leading asymptotic behavior of $q$ and $\Delta \chi_{l}$ also the next-to-leading asymptotic coefficient of the Edwards-Anderson parameter.

To derive the asymptotic form of equations (5) for the geometric parameters $m_{l}$ is more complicated than it was to obtain equations (9) and (10). For the geometric parameters we will need all the coefficients $a_{i j}^{(l)}$ from Appendix A, but those at the highest power $x^{6}$ only for $m_{l}=0$, since $m_{l} \sim x^{2}$. In fact, we have to expand free energy to the order $x^{8}$, but the highest-order terms cancel each other due to subtraction in the numerator on the right-hand side of Eq (5). After some effort we arrive at

$$
\begin{gathered}
\left(\beta^{2}-1\right)\left[\Delta \chi_{l}+2 Q_{l+1}\right] m_{l}=\frac{\beta^{4}}{3} m_{l}\left\{\left[6 \Delta \chi_{l}^{2}+18 \Delta \chi_{l} Q_{l+1}+12 Q_{l+1}^{2}+6\left(\Delta \chi_{l}+2 Q_{l+1}\right) X_{l-1}-6\left(\Delta \chi_{l}+2 Q_{l+1}\right) M_{l-1}\right.\right. \\
\left.-4\left(\Delta \chi_{l}+3 Q_{l+1}\right) m_{l} \Delta \chi_{l}-6 \sum_{i=l+1}^{K} m_{i} \Delta \chi_{i}\left(2 Q_{i+1}+\Delta \chi_{i}\right)\right]-\beta^{2}\left[34 Q_{l+1}^{3}+81 Q_{l+1}^{2} \Delta \chi_{l}+64 Q_{l+1} \Delta \chi_{l}^{2}+16 \Delta \chi_{l}^{3}\right. \\
\left.\left.+10\left(3 \Delta \chi_{l}^{2}+9 Q_{l+1} \Delta \chi_{l}+6 Q_{l+1}^{2}\right) X_{l-1}+3\left(\Delta \chi_{l}+2 Q_{l+1}\right)\left[2 X_{l-1}^{2}+3 \sum_{i=1}^{l-1} \Delta \chi_{i}\left(2 X_{i-1}+\Delta \chi_{i}\right)\right]\right]\right\}, \quad(11)
\end{gathered}
$$

where we denoted $Q_{l+1} \equiv Q-X_{l}$. Also here we had to keep first three orders in the expansion of the righthand side of Eq. (5). In the above asymptotic form we already used the fact that also the geometric parameters at $h=0$ are small near the critical temperature and are of the same order as $q$ and $\Delta \chi_{l}$.

Equations (91)-(11) define the asymptotic behavior of the hierarchical solution near the critical temperature at zero magnetic field. From these equations we are able to determine the leading asymptotic behavior of all the order parameters for an arbitrary number of hierarchies $K$ together with the next-to-leading order of the EdwardsAnderson parameter $Q$. All equations are homogeneous in the respective variable, hence a trivial solution (paramagnetic phase) is correctly included. The asymptotic equations are cubic in the physical order parameters and quadratic in the geometric ones. Such a system of coupled algebraic equations is not generally solvable. We, however, show that a nontrivial solution in the physical sector can always be found. 


\section{NONTRIVIAL SOLUTION OF THE ASYMPTOTIC EQUATIONS}

We now expand the dynamical variables in a small parameter measuring the distance from the critical point in the spin-glass phase $\theta=1-T / T_{c}$. We have to keep only the leading asymptotic order and for the EdwardsAnderson parameter we have to add the next-to-leading order. Not to introduce cumbersome further indexing or new parameters we relabel in this chapter the order parameters as follow $q \rightarrow q \theta, \Delta \chi_{l} \rightarrow \chi_{l} \theta, m_{l} \rightarrow m_{l} \theta$, $Q \rightarrow Q \theta+Q^{\prime} \theta^{2}$. We expand the asymptotic equations in powers of $\theta$ and all expansion coefficients (up to $\theta^{3}$ ) from the left-hand side must equal those from the right-hand side.

Excluding the trivial solution from Eqs. (9)-(11) the first term on the right-hand side defines the critical temperature. From the second one in Eqs. (9) and (10) we obtain

$$
Q=1
$$

Notice that the Edwards-Anderson parameter in the leading order is defined exactly by the replica-symmetric solution $(K=0)$.

The first nontrivial terms contributing to the order parameters of the hierarchical solution are the third ones. The corresponding coefficients at $\theta^{3}$ of Eqs. (9) and (10) are

$$
\begin{gathered}
0=1-3 Q^{\prime}+X_{K}^{2}-2 X_{K}+3 M_{K} \\
0=-6 Q^{\prime}+2 \chi_{l}^{2}+6\left(1-X_{l-1}\right)\left(1-X_{l-1}-\chi_{l}\right) \\
+m_{l} \chi_{l}+6 M_{l-1} .
\end{gathered}
$$

We use the former equation to exclude the next-toleading coefficient $Q^{\prime}$ of the Edwards-Anderson parame- ter, that is

$$
Q^{\prime}=\frac{1}{3}\left[1-X_{K}\left(2-X_{K}\right)+3 M_{K}\right]
$$

Using this result in Eq. (14) we obtain a recursive relation

$$
\begin{gathered}
m_{l} \chi_{l}=\frac{2}{3}\left[2+X_{K}\left(2-X_{K}\right)+\chi_{l}^{2}-3 \chi_{l}\left(1-X_{l-1}\right)\right. \\
\left.-3 X_{l-1}\left(2-X_{l-1}\right)\right]-2 \sum_{i=l+1}^{K} m_{i} \chi_{i} .
\end{gathered}
$$

An explicit solution to this set of recursive equations reads

$$
\begin{gathered}
m_{l} \chi_{l}=\frac{2}{3} \sum_{i=0}^{K-l}\left(2-\delta_{i, 0}\right)(-1)^{i}\left[2+X_{K}\left(2-X_{K}\right)+\chi_{l+i}^{2}\right. \\
\left.-3 \chi_{l+i}\left(1-X_{l+i-1}\right)-3 X_{l+i-1}\left(2-X_{l+i-1}\right)\right]
\end{gathered}
$$

We have now to exclude the geometric parameters $m_{l}$ from Eq. (16). To this purpose we use Eq. (17) in the asymptotic expansion of Eq. (11). We then obtain another set of recursive equations

$$
\begin{gathered}
m_{l} \chi_{l}^{2}=\frac{1}{2}\left(2-2 X_{l-1}-\chi_{l}\right)\left[2 X_{K}\left(2-X_{K}\right)\right. \\
-\chi_{l}\left(2-2 X_{l-1}-\chi_{l}\right)-X_{l-1}\left(4+7 X_{l-1}\right) \\
\left.+9 \sum_{i=1}^{l-1} \chi_{i}\left(2 X_{l-1}+\chi_{i}\right)\right]-3 \sum_{i=l+1}^{K} m_{i} \chi_{i}\left[2\left(X_{i}-X_{l}\right)\right. \\
\left.-\left(\chi_{i}-\chi_{l}\right)\right]
\end{gathered}
$$

Combing Eq. (17) and Eq. (18) we end up with a set of equations with only the leading-order asymptotic coefficients at the differences of the overlap susceptibilities $\chi_{l}$ :

$$
\begin{aligned}
& \frac{4}{3} \chi_{l} \sum_{i=0}^{K-l}\left(2-\delta_{i, 0}\right)(-1)^{i}\left[2+X_{K}\left(2-X_{K}\right)+\chi_{l+i}^{2}-3 \chi_{l+i}\left(1-X_{l+i-1}\right)-3 X_{l+i-1}\left(2-X_{l+i-1}\right)\right] \\
& \quad=\left(2-2 X_{l-1}-\chi_{l}\right)\left[2 X_{K}\left(2-X_{K}\right)-\chi_{l}\left(2-2 X_{l-1}-\chi_{l}\right)-X_{l-1}\left(4+7 X_{l-1}\right)+9 \sum_{i=1}^{l-1} \chi_{i}\left(2 X_{l-1}+\chi_{i}\right)\right] \\
& \quad-4 \sum_{i=l+1}^{K}\left[2\left(X_{i}-X_{l}\right)-\left(\chi_{i}-\chi_{l}\right)\right] \sum_{j=0}^{K-i}\left(2-\delta_{j, 0}\right)(-1)^{j}\left[2+X_{K}\left(2-X_{K}\right)+\chi_{l+i}^{2}-3 \chi_{j+i}\left(1-X_{j+i-1}\right)\right. \\
& \left.-3 X_{j+i-1}\left(2-X_{j+i-1}\right)\right]
\end{aligned}
$$

The set of equations for $\chi_{l}, l=1,2, \ldots, K$ looks rather complicated but we can successively express the susceptibilities $\chi_{l}$ via their sums $X_{l-1}$.
Putting $l=K$ in Eq. (19) we obtain a simple cubic equation for $\chi_{K}$ :

$$
0=\chi_{K}\left(2-2 X_{K-1}-\chi_{K}\right)\left(2-2 X_{K-1}-3 \chi_{K}\right) .
$$


One solution is trivial, $\chi_{K}=0$, one is unphysical, $\chi_{K}=$ $2\left(1-X_{K-1}\right)$. The only physically acceptable solution in the spin-glass phase is $\chi_{K}=2\left(1-X_{K-1}\right) / 3$.

Next we use this physical solution in Eq. (19) for $l=$ $K-1$. We again can factorize this equation for $\chi_{K-1}$ to a product

$$
0=\chi_{K-1}\left(2-2 X_{K-2}-\chi_{K-1}\right)\left(2-2 X_{K-2}-5 \chi_{K-1}\right) .
$$

The only physical solution is $\chi_{K-1}=2\left(1-X_{K-2}\right) / 5$.

We go on with this successive substitutions and derive a generic physical solution

$$
\chi_{K-l}=\frac{2}{2 l+3}\left(1-X_{K-l-1}\right) .
$$

Realizing that $X_{0}=0$ we obtain an explicit solution for all coefficients $\chi_{l}, l=1,2, \ldots, K$.

\section{PROPERTIES OF THE ASYMPTOTIC SOLUTION AND THE CONTINUOUS LIMIT}

After finding an explicit expression for the leading asymptotic terms of the local overlap susceptibilities we can recover the leading-order (in some case also the nextto-leading-order asymptotic) coefficients for all physical quantities of interest. We now use solution (22) in Eqs. (17), (15) and (12) to obtain an explicit asymptotic form of the order parameters:

$$
\begin{aligned}
\Delta \chi_{l}^{K} & \doteq \frac{2}{2 K+1} \theta, \\
m_{l}^{K} & \doteq \frac{4(K-l+1)}{2 K+1} \theta \\
q^{K} & \doteq \frac{1}{2 K+1} \theta
\end{aligned}
$$

and the first two leading orders of the Edwards-Anderson parameter

$$
Q^{K} \doteq \theta+\frac{12 K(K+1)+1}{3(2 K+1)^{2}} \theta^{2} .
$$

We returned to the superscript explicitly denoting the dependence of the order parameters on the number of hierarchies.

First thing we can observe is that both differences $\Delta \chi_{l}^{K}$ and $\Delta m_{l}^{K}=m_{l-1}-m_{l}, m_{0}=1$, do not depend on the hierarchy index $l$. We have $\Delta m_{l}^{K}=2 \Delta \chi_{l}^{K}=4 /(2 K+1)$. It means that if all solutions with finite hierarchical levels are unstable we have to choose the number of hierarchies infinite. The discrete RSB scheme then converges to a continuous theory with either $\Delta \chi^{K}$ or $\Delta m^{K}$ as infinitesimal differentials. The ratio of the two differentials is in the leading order near the critical temperature constant, $\Delta \chi^{K} / \Delta m^{K} \doteq 1 / 2$. This ratio is even independent of the total number of hierarchies $K$.

Before we investigate stability of the solution with $K$ hierarchies we evaluate other physical quantities of interest with the aid of the asymptotic form of the order parameters to see how they depend on the number of hierarchies. With Eqs. (23) we are able to evaluate the leading deviation beyond the paramagnetic solution. We start with the thermal local susceptibility $\chi_{T}$ that in the spin-glass phase does not obey the single-state Fischer relation, $\chi_{T}=\beta(1-Q)$. 14] In the RSB solution we have

$$
\chi_{T}=\beta\left(1-Q+\sum_{l=1}^{K} m_{l} \Delta \chi_{l}\right) \doteq 1-\frac{\theta^{2}}{3(2 K+1)^{2}} .
$$

We see that in the limit $K \rightarrow \infty$ the local susceptibility seems to be a constant below the critical temperature.

Another physical quantity of interest is the density of internal energy that behaves near the critical temperature in the following manner

$$
\begin{aligned}
u & =\frac{\partial}{\partial \beta}(\beta f)=-\frac{\beta}{2}\left(1-Q^{2}\right) \\
& -\frac{\beta}{2} \sum_{l=1}^{K} m_{l} \Delta \chi_{l}\left(2 Q_{l}-\Delta \chi_{l}\right) \doteq-\frac{1}{2}-\frac{\theta}{2}+\frac{\theta^{3}}{3} .
\end{aligned}
$$

The first two terms in the temperature behavior of the internal energy are from the paramagnetic solution (trivial order parameters). The third term is fully determined by the SK solution, since it does not depend on the number of hierarchical levels used.

The last quantity the asymptotic behavior of which we evaluate near the critical temperature is free energy. We do not need to expand the free energy directly, since we can use the asymptotic expansion for the internal energy and the defining equation relating the two quantities from Eq. (25). It can be rewritten to a more suitable form using the expansion parameter $\theta: u=f+(1-\theta) \partial f / \partial \theta$. Using this equation and the asymptotic result from Eq. (25) we easily find

$$
f \doteq-\ln 2-\frac{\theta}{2}(2 \ln 2+1)-\frac{\theta^{2}}{4}-\frac{\theta^{3}}{12}+\frac{\theta^{4}}{24} .
$$

We can see that the leading order asymptotic terms in the order parameters breaking the replica symmetry contribute to the density of free energy only in the fifth order of the deviation from the critical temperature. This order cannot, however, be determined exactly from the leading asymptotic form of the stationarity equations for the order parameters.

We can, nevertheless, improve upon the precision of the asymptotic expansion of free energy and consequently of internal energy without increasing the precision of the order parameters. We can apply the asymptotic expansion in $\theta$ directly to the functional of free energy. We then are able to expand free energy at the saddle point to the order $O\left(\theta^{5}\right)$ and internal energy to $O\left(\theta^{4}\right)$. It appears that the next-to-leading asymptotic contributions to the order parameters cancel each other in free energy up to the order $O\left(\theta^{5}\right)$. Using the asymptotic form of the interacting part of free energy from Eq. (B1) in Eq. (3) 
together with the asymptotic solutions for the order parameters we end up with corrections to the paramagnetic solution of free energy

$$
\Delta f \doteq\left(\frac{1}{6} \theta^{3}+\frac{7}{24} \theta^{4}+\frac{29}{120} \theta^{5}\right)-\frac{1}{360} \theta^{5}\left(\frac{1}{K}\right)^{4}
$$

and of internal energy

$$
\Delta u \doteq\left(\frac{1}{2} \theta^{2}+\frac{5}{6} \theta^{3}+\frac{1}{3} \theta^{4}\right)-\frac{1}{72} \theta^{4}\left(\frac{1}{K}\right)^{4} .
$$

The number of hierarchies $K$ is a free parameter in the general hierarchical solution we analyzed. To make the solution unique we have to decide what choice of this parameter leads to the exact solution. Generally, the number of hierarchies in free energy (3) is the minimal one for which all stability conditions from Eq. (6) are satisfied. We have $K+1$ stability conditions for a given $K$. In the first two leading asymptotic orders they can be rewritten with the aid of the order parameters as

$$
\begin{aligned}
& \Lambda_{l} \doteq 1 \\
& -\beta^{2}\left[1-2 Q+2 M_{l}+X_{l}^{2}+2 Q_{l+1} X_{l}+3 Q_{l+1}^{2}\right] .
\end{aligned}
$$

Using the relation between quantities $Q_{l+1}$ and $X_{l}$ and the expansion of the order parameters to the leading order and the Edwards-Anderson parameter to the nextto-leading order we obtain

$$
\begin{aligned}
& \Lambda_{l} \doteq-2 \theta(1-Q) \\
& -2 \theta^{2}\left[\left(1-X_{l}\right)^{2}-\frac{1}{3}\left(1-X_{K}\right)^{2}+M_{l}-M_{K}\right] .
\end{aligned}
$$

It is clear from the solution from Eq. (23) that the linear term on the right-hand side of Eq. (30) vanishes. The first nontrivial temperature-dependent term then reads 15

$$
\Lambda_{l}=-\frac{4}{3} \frac{\theta^{2}}{(2 K+1)^{2}} .
$$

All the stability conditions collapse in the leading asymptotic order to a single expression independent of the hierarchical index $l$. Since the stability parameters $\Lambda_{l}$ are negative at any $K$, we conclude that no finite number of hierarchies in Eq. (3) is able to produce a physical solution with no negative values for the stability parameters. Since $\lim _{K \rightarrow \infty} \Lambda_{l}=0$, only the solution with infinite-many hierarchical levels is physically acceptable. The RSB solution with infinite many hierarchies is then marginally stable.

We can see from the asymptotic solution (23) that the limit of infinite-many hierarchies leads to a continuous limit with infinitesimal differentials $\Delta \chi_{l}$ or $\Delta m_{l}$. The continuous limit then results in simplifications of the hierarchical free energy and the corresponding equations of motion. First, all higher than linear powers of the difference $\Delta \chi_{l}$ (for the fixed index $l$ ) vanish from the continuous limit. With this simplification in mind we can significantly reduce the set of equations (19) for the leading-order coefficients of the overlap susceptibilities We assign continuous quantities to the following discrete variables: $X_{l} \rightarrow x, m_{l} \rightarrow m(x), X_{K} \rightarrow x_{\max }$. Equation (19) then reduces to

$$
\begin{aligned}
3 \int_{x}^{x_{\max }} & d y(y-x) m(y) \\
& =(1-x)\left[x_{\max }\left(2-x_{\max }\right)-x(2-x)\right] .
\end{aligned}
$$

We know that $x_{\max }=1-q$. We can now solve Eq. (32) independently of the discrete approximations. We denote $M(x)=\int_{0}^{x} \operatorname{dym}(y)$ and $M=M(1)$ and rewrite Eq. (32) to

$$
\begin{aligned}
3(M-M(x))=x_{\max }\left(2-x_{\max }\right)- & x(2-x) \\
+ & 2(1-x)^{2} .
\end{aligned}
$$

From continuity of the function $M(x)$ we obtain $x_{\max }=$ 1 and hence $q=0$. A derivative of this equation with respect to $x$ leads to an explicit representation

$$
m(x)=2(1-x) .
$$

Finally, using the continuous version of Eq. (15) we derive the next-to-leading asymptotic term of the EdwardsAnderson order parameter $Q^{\prime}=\int_{0}^{1} d x m(x)=M=1$.

The solution obtained from the continuous version of the RSB scheme coincides with the limit of the discrete scheme with infinite number of hierarchies. All the physical quantities, when infinite-many hierarchies are needed to reach a stable solution, can hence be derived directly from the continuous RSB scheme. The only information we lose in the continuous solution is the rate with which the continuous limit is approached.

\section{CONCLUSIONS}

We studied in this paper the full discrete hierarchy of RSB solutions of the zero-field SK model in the critical region below the transition temperature to the spin-glass phase. We expanded the stationarity equations for all the order parameters to an appropriate order in $\theta=1-T / T_{c}$ so as to find their leading asymptotic behavior. We succeeded in solving the resulting equations and obtained an explicit leading asymptotic behavior of all thermodynamic functions of the RSB solution with an arbitrary number of hierarchies. The number of hierarchies in the RSB solution is treated as a free parameter to be determined from stability conditions. Stability of the RSB scheme with $K$ hierarchies is measured by $K+1$ numbers $\Lambda_{l}, l=0,1, \ldots, K$ from Eq. (6). All these numbers must be nonnegative in a (marginally) stable solution. We explicitly evaluated these numbers with the asymptotic solution for the order parameters and found that any solution with finite-many hierarchies is unstable 
with instability of order $\theta^{2}$. Near the critical temperature all the stability conditions collapse in the first two leading asymptotic orders to a single criterion. With the increasing number of hierarchies the instability parameters decrease and become zero at $K=\infty$. Hence first the infinite-order RSB is marginally stable below the critical temperature.

The most important physical issue we addressed in this paper was the legitimacy of the continuous limit. We proved by explicit calculation that the discrete RSB scheme indeed converges toward the continuous limit with $K \rightarrow \infty$. We found that near the critical temperature both the overlap susceptibilities $\chi_{l}$ and the geometric parameters $m_{l}$ determining the way the replica symmetry is broken are uniformly distributed and their differences $\Delta \chi_{l}$ and $\Delta m_{l}$ become infinitesimal for $K \rightarrow \infty$. Either of these differences can hence be used as a fundamental differential for the continuous limit. Since the differences with the same hierarchy index $l$ appear in the free energy of the continuous limit only linearly, we loose one set of stationarity equations from the discrete version. It appeared more natural to choose $d x=\lim _{K \rightarrow \infty} \Delta \chi_{K}$ as the underlying differential for the continuous formulation of the RSB solution with infinite-many hierarchies. Hence, $\Delta \chi_{l}$ are no longer variational parameters in the continuous limit and only $x_{\max }$, the maximal value for the overlap susceptibility is to be determined from the free energy functional for the continuous limit of the RSB scheme.

The asset of the present construction, however, is not only in that it provides a proof of exactness of the continuous limit for the zero-field SK model near the critical temperature. This conclusion was actually believed to be correct already from the existing less accurate treatments. With our construction we set up a general scheme how to solve the RSB equations with an arbitrary number of hierarchies near the critical point practically for any mean-field spin-glass model. We applied this construction to the case of zero magnetic field in the SK model. But the same scheme can be applied also in the presence of a magnetic field and expand the hierarchical solution around the de Almeida-Thouless line. In this case, however, the geometric order parameters are

no longer small. We can as well apply the expansion scheme to other mean-field spin-glass models. Of particular interest are those where one expects that in certain parameter regions the RSB solutions with a few hierarchies (1RSB) are stable, such as the Potts spin glass. The question that has not yet been answered is whether the instability of the 1RSB solution there leads already to the continuous limit or not. The scheme presented here can conveniently be used to address this problem.

\section{Acknowledgments}

Research on this problem was carried out within a project AVOZ10100520 of the Academy of Sciences of the Czech Republic and supported in part by Grant No. IAA1010307 of the Grant Agency of the Academy of Sciences of the Czech Republic.

\section{APPENDIX A: EXPANSION COEFFICIENTS FOR PARTITION SUM NEAR THE CRITICAL TEMPERATURE}

Here we list the expansion coefficients needed for the asymptotic solution near the critical temperature. We evaluate the coefficients used for the $l$ th partition sum in Eq. (7). We used MATHEMATICA to derive the recursive relations from which we then obtained

$$
\begin{aligned}
& a_{0 i}^{(l)}=a_{0 i} \\
& a_{20}^{(l)}=a_{02} \sum_{i=1}^{l} c_{i}^{2} \\
& a_{22}^{(l)}=6 a_{04} \sum_{i=1}^{l} c_{i}^{2}+2 a_{02}^{2} \sum_{i=1}^{l}\left(m_{i}-1\right) c_{i}^{2} \\
& a_{24}^{(l)}=15 a_{06} \sum_{i=1}^{l} c_{i}^{2}+2 a_{02}\left(4 a_{04}-a_{02}^{2}\right) \sum_{i=1}^{l}\left(m_{i}-1\right) c_{i}^{2}
\end{aligned}
$$

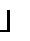

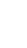

$$
\begin{gathered}
a_{40}^{(l)}=3 a_{04} \sum_{i=1}^{l} c_{i}^{2}\left[2 \sum_{j=1}^{i-1} c_{j}^{2}+c_{i}^{2}\right]+a_{02}^{2} \sum_{i=1}^{l} c_{i}^{2}\left[2 \sum_{j=1}^{i-1}\left(m_{j}-1\right) c_{j}^{2}+\left(m_{i}-1\right) c_{i}^{2}\right] \\
a_{42}^{(l)}=45 a_{06} \sum_{i=1}^{l} c_{i}^{2}\left[2 \sum_{j=1}^{i-1} c_{j}^{2}+c_{i}^{2}\right]+12 a_{02} a_{04} \sum_{i=1}^{l} c_{i}^{2}\left[\left(m_{i}-1\right)\left(3 c_{i}^{2}+2 \sum_{j=1}^{i-1} c_{j}^{2}\right)+4 \sum_{j=1}^{i-1}\left(m_{j}-1\right) c_{j}^{2}\right] \\
+a_{02}^{3} \sum_{i=1}^{l} c_{i}^{2}\left\{\left(m_{i}-1\right)\left[\left(4 m_{i}-11\right) c_{i}^{2}+2 \sum_{j=1}^{i-1}\left(4 m_{j}-5\right) c_{j}^{2}\right]-12 \sum_{j=1}^{i-1}\left(m_{j}-1\right) c_{j}^{2}\right\}
\end{gathered}
$$




$$
\begin{aligned}
a_{60}^{(l)}= & 15 a_{06} \sum_{i=1}^{l} c_{i}^{2}\left[c_{i}^{4}+3 c_{i}^{2} \sum_{j=1}^{i-1} c_{j}^{2}+3 \sum_{j=1}^{i-1} c_{j}^{2}\left(2 \sum_{k=1}^{j-1} c_{k}^{2}+c_{j}^{2}\right)\right]+12 a_{02} a_{04} \sum_{i=1}^{l} c_{i}^{2}\left\{\left(m_{i}-1\right) c_{i}^{2}\left(c_{i}^{2}+\sum_{j=1}^{i-1} c_{j}^{2}\right)\right. \\
& \left.+2 c_{i}^{2} \sum_{j=1}^{i-1}\left(m_{j}-1\right) c_{j}^{2}+\sum_{j=1}^{i-1} c_{j}^{2}\left[\left(m_{j}-1\right)\left(c_{j}^{2}+2 \sum_{k=1}^{j-1} c_{k}^{2}\right)+4 \sum_{k=1}^{j-1}\left(m_{k}-1\right) c_{k}^{2}\right]\right\} \\
+ & a_{02}^{3} \sum_{i=1}^{l} c_{i}^{2}\left\{\frac{1}{3}\left(m_{i}-1\right) c_{i}^{2}\left[\left(4 m_{i}-11\right) c_{i}^{2}+3 \sum_{j=1}^{i-1}\left(4 m_{j}-5\right) c_{j}^{2}\right]-6 c_{i}^{2} \sum_{j=1}^{i-1}\left(m_{j}-1\right) c_{j}^{2}\right. \\
& \left.-12 \sum_{j=1}^{i-1} c_{j}^{2} \sum_{k=1}^{j-1}\left(m_{k}-1\right) c_{k}^{2}+\sum_{j=1}^{i-1}\left(m_{j}-1\right) c_{j}^{2}\left[\left(4 m_{j}-11\right) c_{j}^{2}+2 \sum_{k=1}^{j-1}\left(4 m_{k}-5\right) c_{k}^{2}\right]\right\}
\end{aligned}
$$

The initial coefficients are determined by the hyperbolic cosine in $Z_{0}$ and read $a_{00}=1, a_{02}=1 / 2, a_{04}=1 / 24$, and $a_{06}=1 / 720$.

\section{APPENDIX B: DIRECT ASYMPTOTIC EXPANSION OF FREE ENERGY}

Instead of expanding the stationarity equations we can expand directly free energy near the critical point. The advantage of such an expansion is a possibility to go to higher orders of the small expansion parameter more easier than in the case of stationarity equations. The drawback of a direct expansion of free energy is that when building up the corresponding stationarity equations we have to vary w.r.t. small parameters and we change the order of the expansion to which we keep all terms exact.
It then may happen that different stationarity equations are evaluated not in the same order of the expansion parameter.

The expansion of free energy is achieved in the same manner in which we treated the partition sum with the expansion coefficients from Appendix A. Using the program MATHEMATICA we were able to go to the fifth order in $\theta$. In this expansion we, however took into consideration the fact that the geometric parameters $m_{l} \propto \theta$ and neglected all combinations of the order parameters of order $O\left(\theta^{6}\right)$. It appears that the next-to-leading order contributions to the order parameters actually contribute to free energy nontrivially first in the order $\theta^{6}$. It is not manifestly evident but an explicit expansion discloses this feature. If we denote $\mathcal{G}_{K}=\int_{-\infty}^{\infty} \mathcal{D} \eta \ln Z_{K}$ we obtain an exact asymptotic for arbitrary number of hierarchical levels 


$$
\begin{aligned}
& \mathcal{G}_{5}^{K} \equiv \sum_{l=1}^{K}\left\{\frac{31}{15} \Delta \chi_{l}^{5} \beta^{10}+\left(\frac{31 q \beta^{2}}{3}+\frac{4 m_{l}}{3}-\frac{17}{24}\right) \Delta \chi_{l}^{4} \beta^{8}+\left(\frac{62 q^{2} \beta^{4}}{3}-\frac{17 q \beta^{2}}{6}+\frac{16}{3} q m_{l} \beta^{2}+\frac{m_{l}^{2}}{6}-\frac{m_{l}}{2}+\frac{1}{3}\right) \Delta \chi_{l}^{3} \beta^{6}\right. \\
& +\left(\frac{62 q^{3} \beta^{6}}{3}-\frac{17 q^{2} \beta^{4}}{4}+\frac{27}{4} q^{2} m_{l} \beta^{4}+\frac{1}{2} q m_{l}^{2} \beta^{2}+q \beta^{2}-\frac{3}{2} q m_{l} \beta^{2}+\frac{m_{l}}{4}-\frac{1}{4}\right) \Delta \chi_{l}^{2} \beta^{4} \\
& \left.+\left(\frac{1}{2}\left(\frac{62 q^{4} \beta^{8}}{3}-\frac{17 q^{3} \beta^{6}}{3}+2 q^{2} \beta^{4}-q \beta^{2}\right)+\frac{1}{2}\left(\frac{17 q^{3} \beta^{6}}{3}-2 q^{2} \beta^{4}+q \beta^{2}\right) m_{l}+\frac{1}{2}\right) \Delta \chi_{l} \beta^{2}\right\} \\
& +\sum_{l=1}^{K} \sum_{j=l+1}^{K}\left\{\frac{62}{3}\left(\Delta \chi_{l}^{2} \Delta \chi_{j}^{3}+\Delta \chi_{l}^{3} \Delta \chi_{j}^{2}\right) \beta^{10}+\frac{31}{3}\left(\Delta \chi_{l} \Delta \chi_{j}^{4}+\Delta \chi_{l}^{4} \Delta \chi_{j}\right) \beta^{10}+\left(\frac{124 q \beta^{2}}{3}+\frac{16 m_{l}}{3}-\frac{17}{6}\right) \Delta \chi_{j} \Delta \chi_{l}^{3} \beta^{8}\right. \\
& +\left(62 q \beta^{2}+\frac{5 m_{j}}{4}+\frac{27 m_{l}}{4}-\frac{17}{4}\right) \Delta \chi_{j}^{2} \Delta \chi_{l}^{2} \beta^{8}+\left(\frac{124 q \beta^{2}}{3}+\frac{5 m_{j}}{2}+\frac{17 m_{l}}{6}-\frac{17}{6}\right) \Delta \chi_{j}^{3} \Delta \chi_{l} \beta^{8} \\
& +\left(62 q^{2} \beta^{4}-\frac{17 q \beta^{2}}{2}+\frac{5}{2} q m_{j} \beta^{2}+\frac{27}{2} q m_{l} \beta^{2}+\frac{m_{l}^{2}}{2}-\frac{3 m_{l}}{2}+1\right) \Delta \chi_{j} \Delta \chi_{l}^{2} \beta^{6} \\
& +\left(62 q^{2} \beta^{4}-\frac{17 q \beta^{2}}{2}+\frac{15}{2} q m_{j} \beta^{2}+\frac{17}{2} q m_{l} \beta^{2}-\frac{m_{j}}{2}+\frac{m_{j} m_{l}}{2}-m_{l}+1\right) \Delta \chi_{j}^{2} \Delta \chi_{l} \beta^{6} \\
& \left.+\left(\frac{124 q^{3} \beta^{6}}{3}-\frac{17 q^{2} \beta^{4}}{2}+5 q^{2} m_{j} \beta^{4}+\frac{17}{2} q^{2} m_{l} \beta^{4}+2 q \beta^{2}-q m_{j} \beta^{2}-2 q m_{l} \beta^{2}+q m_{j} m_{l} \beta^{2}+\frac{m_{l}}{2}-\frac{1}{2}\right) \Delta \chi_{j} \Delta \chi_{l} \beta^{4}\right\} \\
& +\sum_{l=1}^{K} \sum_{j=l+1}^{K} \sum_{i=j+1}^{K}\left\{62\left(\Delta \chi_{i} \Delta \chi_{l}^{2} \Delta \chi_{j}^{2}+\Delta \chi_{i}^{2} \Delta \chi_{l} \Delta \chi_{j}^{2}+\Delta \chi_{i}^{2} \Delta \chi_{l}^{2} \Delta \chi_{j}\right) \beta^{10}\right. \\
& +\frac{124}{3}\left(\Delta \chi_{j} \Delta \chi_{l} \Delta \chi_{i}^{3}+\Delta \chi_{j} \Delta \chi_{l}^{3} \Delta \chi_{i}+\Delta \chi_{j}^{3} \Delta \chi_{l} \Delta \chi_{i}\right) \beta^{10}+\left(124 q \beta^{2}+\frac{5 m_{j}}{2}+\frac{27 m_{l}}{2}-\frac{17}{2}\right) \Delta \chi_{i} \Delta \chi_{j} \Delta \chi_{l}^{2} \beta^{8} \\
& +\left(124 q \beta^{2}+\frac{15 m_{j}}{2}+\frac{17 m_{l}}{2}-\frac{17}{2}\right) \Delta \chi_{i} \Delta \chi_{j}^{2} \Delta \chi_{l} \beta^{8}+\left(124 q \beta^{2}+\frac{5 m_{i}}{2}+5 m_{j}+\frac{17 m_{l}}{2}-\frac{17}{2}\right) \Delta \chi_{i}^{2} \Delta \chi_{j} \Delta \chi_{l} \beta^{8} \\
& \left.+\left(124 q^{2} \beta^{4}-17 q \beta^{2}+5 q m_{i} \beta^{2}+10 q m_{j} \beta^{2}+17 q m_{l} \beta^{2}-m_{j}+m_{j} m_{l}-2 m_{l}+2\right) \Delta \chi_{i} \Delta \chi_{j} \Delta \chi_{l} \beta^{6}\right\} \\
& +\sum_{l=1}^{K} \sum_{j=l+1}^{K} \sum_{i=j+1}^{K} \sum_{k=i+1}^{K}\left\{124\left(\Delta \chi_{j} \Delta \chi_{k} \Delta \chi_{l} \Delta \chi_{i}^{2}+\Delta \chi_{j} \Delta \chi_{k} \Delta \chi_{l}^{2} \Delta \chi_{i}+\Delta \chi_{j} \Delta \chi_{k}^{2} \Delta \chi_{l} \Delta \chi_{i}+\Delta \chi_{j}^{2} \Delta \chi_{k} \Delta \chi_{l} \Delta \chi_{i}\right) \beta^{10}\right. \\
& \left.+\left(248 q \beta^{2}+5 m_{i}+10 m_{j}+17 m_{l}-17\right) \Delta \chi_{i} \Delta \chi_{j} \Delta \chi_{k} \Delta \chi_{l} \beta^{8}\right\} \\
& +\sum_{l=1}^{K} \sum_{j=l+1}^{K} \sum_{i=j+1}^{K} \sum_{k=i+1}^{K} \sum_{p=k+1}^{K} 248 \beta^{10} \Delta \chi_{i} \Delta \chi_{j} \Delta \chi_{k} \Delta \chi_{l} \Delta \chi_{p}
\end{aligned}
$$

Although the interacting part of free energy $\mathcal{G}_{K}$ is of one order higher than the partition sum we used in the stationarity equations, we are unable to evaluate the nextto-leading order of the asymptotic solutions for the order parameters. To calculate the second asymptotic coefficients of the order parameters, in particular the geometric ones, one had to know the complete form of free energy to order $O\left(\theta^{6}\right)$.
[1] G. Parisi, J. Phys. A 13, 1101, (1980).

[2] G. Parisi, J. Phys. A 13, L115, (1980).

[3] G. Parisi, Phys. Rev. Lett. 43, 1754, (1979).

[4] C. De Dominicis and I. Kondor, Phys. Rev. B27, 606 (1983).

[5] I. Kondor, J. Phys. A.: Math. Gen. 16, L127 (1980).

[6] H. J. Sommers, J. Physique Lett. 46 L779, (1985).

[7] A. Crisanti and T. Rizzo, Phys. Rev. E65, 046137 (2002).

[8] B. Derrida, Phys. Rev. Lett. 45, 79 (1980).
[9] E. Gardner, Nucl. Phys. B240, 747 (1985).

[10] D. Elderfield and D. Sherrington, J. Phys. C16, L497 (1983).

[11] V. Janiš, Phys. Rev. B71, 214403 (2005).

[12] V. Dotsenko, Introduction to the Replica Theory of Disordered Statistical Systems, Cambridge University Press, (Cambridge 2001).

[13] T. Aspelmeier and M. Moore, arXiv:cond-mat/0211707.

[14] K. H. Fischer and J. A. Hertz, Spin Glasses (Cambridge 
University Press, Cambridge 1991).

[15] Note that a similar result on instability of $K \mathrm{RSB}$ solution near the critical model was derived within a truncated model in C. De Dominicis and P. Di Francesco, arXiv:cond-mat/0301066. This calculation was done with only the simplest replica-symmetry-breaking term ( $\sum_{i j k l} \Delta \chi_{i} \Delta \chi_{j} \Delta \chi_{k} \Delta \chi_{l}$ and linear in $m_{l}, \Delta \chi_{l}$ for fixed $\left.l\right)$ in the free energy introduced in Ref. 3]. The asymptotic behavior of this truncated model near the critical temperature is qualitatively correct but the numerical value of the instability (negative value of the spin-glass susceptibility) differs from the exact one in Eq. (31). 